\title{
What do we really know about cold nuclear matter effects?
}

\section{(for heavy quarkonia)}

\author{
L.A. Linden Levy ${ }^{\mathrm{a}}$ \\ for the PHENIX Collaboration \\ University of Colorado, Boulder, CO 80309-0390, USA
}

Received: 30 September 2008 / Revised: 26 January 2009 / Published online: 3 March 2009

(C) Springer-Verlag / Società Italiana di Fisica 2009

\begin{abstract}
The measurement of charmonium suppression in relativistic heavy ion collisions is posited to be an unambiguous probe of the properties of the strongly interacting quark gluon plasma (sQGP). In hot and dense QCD matter Debye color screening prevents charm and anti-charm quark pairs from forming $J / \psi$ mesons if the screening radius is smaller than the binding radius. However, one must have a clear understanding of the expected suppression in normal density QCD matter before interpreting any additional anomalous suppression. The PHENIX experiment has measured $J / \psi$ production from colliding proton + proton and deuteron + gold beams at $200 \mathrm{GeV}$ from the relativistic heavy ion collider (RHIC). The deuteron + gold data can be compared to the proton + proton baseline in order to establish the typical suppression in cold nuclear matter (CNM). For PHENIX, a suppression of $J / \psi$ in cold nuclear matter is observed as one goes forward in rapidity (in the deuterongoing direction), corresponding to a region more sensitive to initial state low- $x$ gluons in the gold nucleus. These results can be convoluted with the nuclear-environment-modified parton distribution functions, extracted from deep inelastic scattering (DIS) and Drell-Yan (DY) data, in order to estimate the $J / \psi$ break up cross section in cold nuclear matter. One can also use a data driven method that does not rely on the assumption of the production mechanism, or PDF parameterization, to extrapolate to the heavy ion collision case. At this time both the predictions for CNM effect suppression in heavy ion collisions are somewhat ambiguous. Future results using the data acquired by the PHENIX experiment in run-6 $(\mathrm{p}+\mathrm{p})$ and run-8 $(\mathrm{d}+\mathrm{Au})$ will be vital for our understanding. These data, which are in the process of being analyzed, will provide a needed improvement in the statistical and systematic precision of constraints for CNM effects. These constraints must be improved in order to make firm
\end{abstract}

a e-mail: lindenle@ colorado.edu conclusions concerning additional hot nuclear matter charmonium suppression in the sQGP.

PACS 25.75.-q $\cdot 12.38 . \mathrm{Mh} \cdot 13.20 . \mathrm{Gd}$

\section{Why the $J / \psi$ ?}

The heavy nature of charmonium $(c \bar{c})$ allows one to apply potential models in non-relativistic quantum mechanics to calculate the mesons binding radius. Originally it was predicted that the modification of the heavy quark pairs potential in the hot dense matter created in heavy ion collisions would cause the pair to become uncorrelated due to color charge screening. This modification of the pair potential via a Debye mass term leads to charmonium suppression [1] when compared to a binary collision scaled proton-proton $(p+p)$ reference. Due to the different binding energies for the different charmonium states one could gain access to the temperature of the medium. At RHIC the suppression of the lowest energy charmonium state, the $J / \psi$ meson, has been measured in gold-gold $(\mathrm{Au}+\mathrm{Au})$ and copper-copper $(\mathrm{Cu}-\mathrm{Cu})$ collisions in $\sqrt{s}_{\mathrm{NN}}=200 \mathrm{GeV}$ collisions.

In order to interpret these data one must remove any effects that occur in normal density cold nuclear matter (CNM). One such effect is the modification of parton distribution functions in a nuclear environment $[2,3]$. Another is Cronin enhancement that leads to a hardening of the transverse momentum spectrum of collision products due to multiple scattering. To this end the PHENIX experiment has also measured the nuclear modification present in deuteron + gold $(\mathrm{d}+\mathrm{Au})$ collisions in $\sqrt{s}=200 \mathrm{GeV}$ collision. The $d+A u$ data is used to extrapolate within a Glauber [4] based data driven model to the Au + Au collision case to predict the suppression that would result from CNM effects and search for an anomalous suppression of $J / \psi$ mesons in the sQGP. 


\section{Nuclear modification factor}

In order to calculate the nuclear modification factor $R_{\mathrm{dAu}}$ the invariant yield of $J / \psi$ mesons from $\mathrm{d}+\mathrm{Au}$ and $\mathrm{p}+\mathrm{p}$ collisions must be measured. Equation (2.1) shows the nuclear modification as a function of rapidity $(y)$ in a given centrality bin $(i)$ which defines the number of binary collisions $\left(N_{i}^{\text {coll }}\right)$. To ensure that the nuclear modification factor is self-consistent the numerator and denominator should be calculated by applying the same analysis cuts and techniques

$R_{\mathrm{dAu}}(y ; i)=\frac{d N_{i}^{\mathrm{d}+\mathrm{Au}} / d y}{N_{i}^{\mathrm{coll}} d N^{\mathrm{p}+\mathrm{p}} / d y}$.

Recently PHENIX published a new analysis of the 2003 RHIC d + Au data with some improvements [5]. In particular a new $p+p$ reference was used from the RHIC 2005 run. This new baseline has an order of magnitude improvement in statistics over the previous measurement and is also used as a reference for the $\mathrm{Au}+\mathrm{Au}[6]$ and $\mathrm{Cu}+\mathrm{Cu}$ [7] nuclear suppression factors in the data driven extrapolation comparison.

It was also necessary to re-analyze the run- $3 \mathrm{~d}+\mathrm{Au}$ data to ensure that a consistent analysis methodology was applied to both data sets. This re-analysis had the added advantage of two years worth of improvements in the data reconstruction software, signal extraction techniques and understanding of the detector over the previous analysis [8]. The nuclear modification factors from this work are shown as a function of rapidity in Fig. 2.1 and versus centrality in Fig. 2.2.

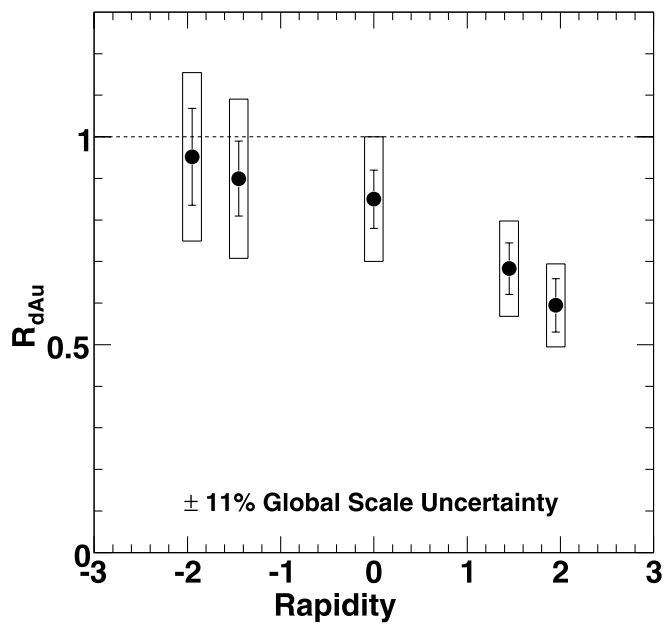

Fig. 2.1 $R_{\mathrm{dAu}}$ versus rapidity for minimum bias collisions. The error bars represent the point to point uncorrelated error. The boxes are the point to point correlated error and the global error is indicated in the figure

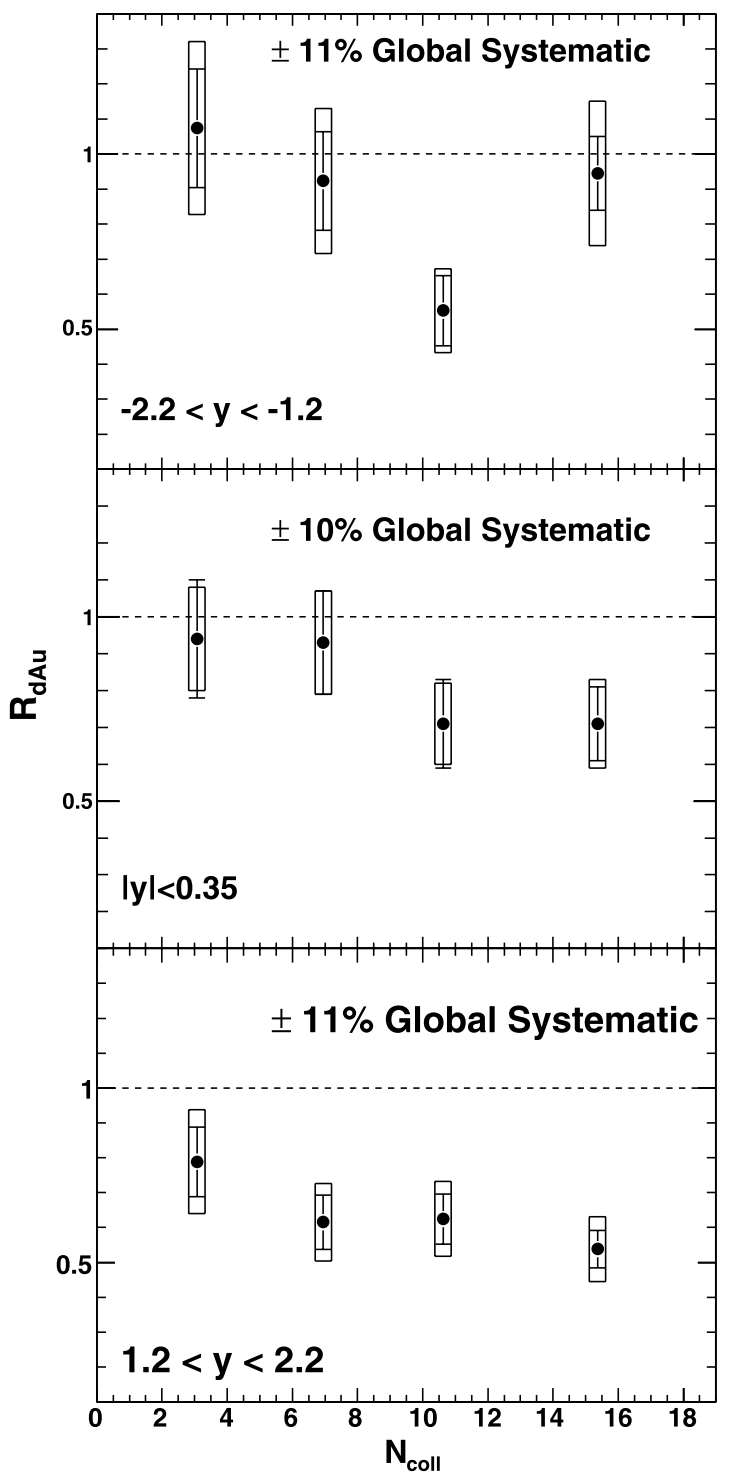

Fig. 2.2 $R_{\mathrm{dAu}}$ versus centrality in three rapidity bins. The error bars represent the point to point uncorrelated error. The boxes are the point to point correlated error and the global error is indicated in the figure

\section{Comparison to shadowing parameterizations}

One obvious, but very model dependant, approach to interpreting the CNM effects seen in the $d+A u$ data relies on nuclear PDF (nPDF) parameterizations. In this approach the $J / \psi$ rapidity and transverse momentum can be related to the momentum fraction of the parton participating in the hard scattering. Using a given nuclear PDF parameterization one can then calculate a nuclear modification factor as a function of rapidity. While the shadowing and anti-shadowing features of nPDFs describe the shape of the data a free parameter termed the "breakup" cross section is employed to account for the overall magnitude of the modification. This parameter is interpreted as the breakup of a correlated $c \bar{c}$ pair while it traverses the remnant of the nucleus. This ap- 


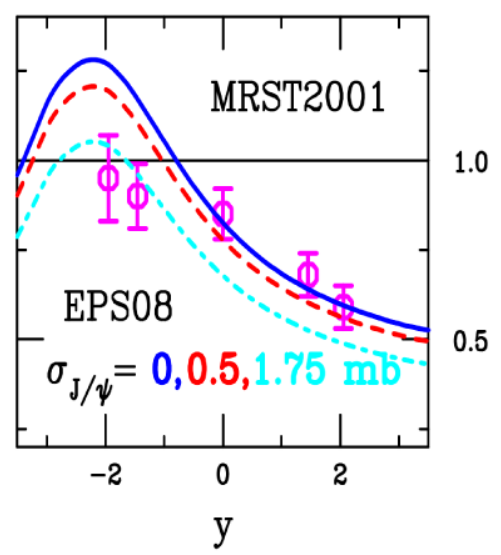

Fig. 3.1 (Color online) PHENIX $R_{\mathrm{dAu}}$ measurement compared to predictions from EPS08 using the intrinsic rapidity $(y)$ to momentum fraction $(x)$ relation (by $\mathrm{R}$. Vogt [9])

proach requires many assumptions, for instance the mapping of the rapidity to parton momentum fraction depends heavily on the assumption of a given production model. In Fig. 3.1 ([9]) the PHENIX measurement is compared to a recent $\mathrm{nPDF}$ parameterization [10] using three possible values for the breakup cross section. It is clear from the figure that the parameterization has more anti-shadowing than the data would allow.

One might believe that the amount of nuclear modification for a PDF should have a dependence on the transverse position in the nucleus. However, one cannot tag the impact parameter in DIS or DY, which are the experimental techniques used to extract these nuclear modified PDFs. Therefore a Glauber [4] based parameterization can be employed in order to include a centrality dependence [11, 12]. In this approach one parameterizes the amount of shadowing as a function of the impact parameter using the local density within the nucleus. The result is a prediction for CNM suppression as a function of rapidity and centrality. Of course in this case as in the minimum bias case one also has the option to include a free parameter for the correlated $c \bar{c}$ breakup cross section.

\section{Data driven method}

Though there is a certain comfort in using models based on measurements to understand the CNM they suffer from unknown uncertainties that are difficult to gauge. It is perhaps better to employ an approach that does not rely on model assumptions. In this approach, termed "data driven", parameterizations for PDFs and a break up cross section are not used to predict the observed CNM effects. Rather one uses the data itself to parameterize a suppression factor in three rapidity ranges and as a function of impact parameter. Here the impact parameter for the $\mathrm{d}+\mathrm{Au}$ collision is measured by the signal in the beam-beam counter in the gold going direction through a full simulation of this detectors response for the signal based on four different centrality classes from a Glauber Monte Carlo [5].

Using the consistently analyzed run-3 d + Au with run-5 $p+p$ baseline one can extract centrality dependant absorption functions in three rapidity ranges as described in [13]. These suppressions factors can be used to describe the expected $\mathrm{Au}+\mathrm{Au} \mathrm{CNM}$ suppression as an average of the convolution (see (4.1)) of the gold $(-y)$ and deuteron $(+y)$ going direction suppression.

$$
R_{\mathrm{AuAu}}=\frac{1}{N_{\mathrm{coll}}} \sum_{i=1}^{N_{\mathrm{coll}}}\left(R_{\mathrm{dAu}}\left(-y, b_{1}^{i}\right) \times R_{\mathrm{dAu}}\left(+y, b_{2}^{i}\right)\right) .
$$

In Fig. 4.1 we show the extrapolation using the data driven method for CNM effects expected in $\mathrm{Au}+\mathrm{Au}$ collisions at $\sqrt{s}=200 \mathrm{GeV}$ [6]. One can see in the top panel of the figure that the mid rapidity suppression is consistent within the uncertainty band with the amount expected from $\mathrm{CNM}$ effects alone. For the forward rapidity the data are not significantly different from the uncertainty band though it is clear that for the most central collisions the difference increases and the data are less consistent then the mid rapidity case. In experimental physics a "discovery" measurement is often quoted at the five sigma level, while this definition may be a bit drastic in this case, the significance of the deviation at forward rapidity for the most central collisions is less than two sigma. Unambiguous evidence for anomalous $J / \psi$ suppression requires more work to reduce the uncertainty band in the projection.

\section{Future measurements}

The current extrapolation of CNM to heavy ion collisions does not allow one to make an overwhelming case for anomalous suppression. At forward rapidity in the $\mathrm{Au}+\mathrm{Au}$ data the measured nuclear modification is near the lower limit of the uncertainty band, however within the uncertainties there is not a statistically significant signal. It is clear that the statistical and systematic errors on the $d+$ Au nuclear modification factor need to be improved.

During the 2008 RHIC d + Au run PHENIX recorded a factor of 30 more statistics compared to $2.4 \mathrm{nb}^{-1}$ collected in 2003. In addition to the new $d+$ Au measurement a new $p+p$ reference set is also available from 2006 that has a factor of three increase over the $2005 \mathrm{p}+\mathrm{p}$ baseline. Using these two new data sets the PHENIX collaboration will extract a more precise prediction for $J / \psi \mathrm{CNM}$ effects in heavy ion collisions. In particular, our current analysis work focuses heavily on reducing the systematic errors resulting 

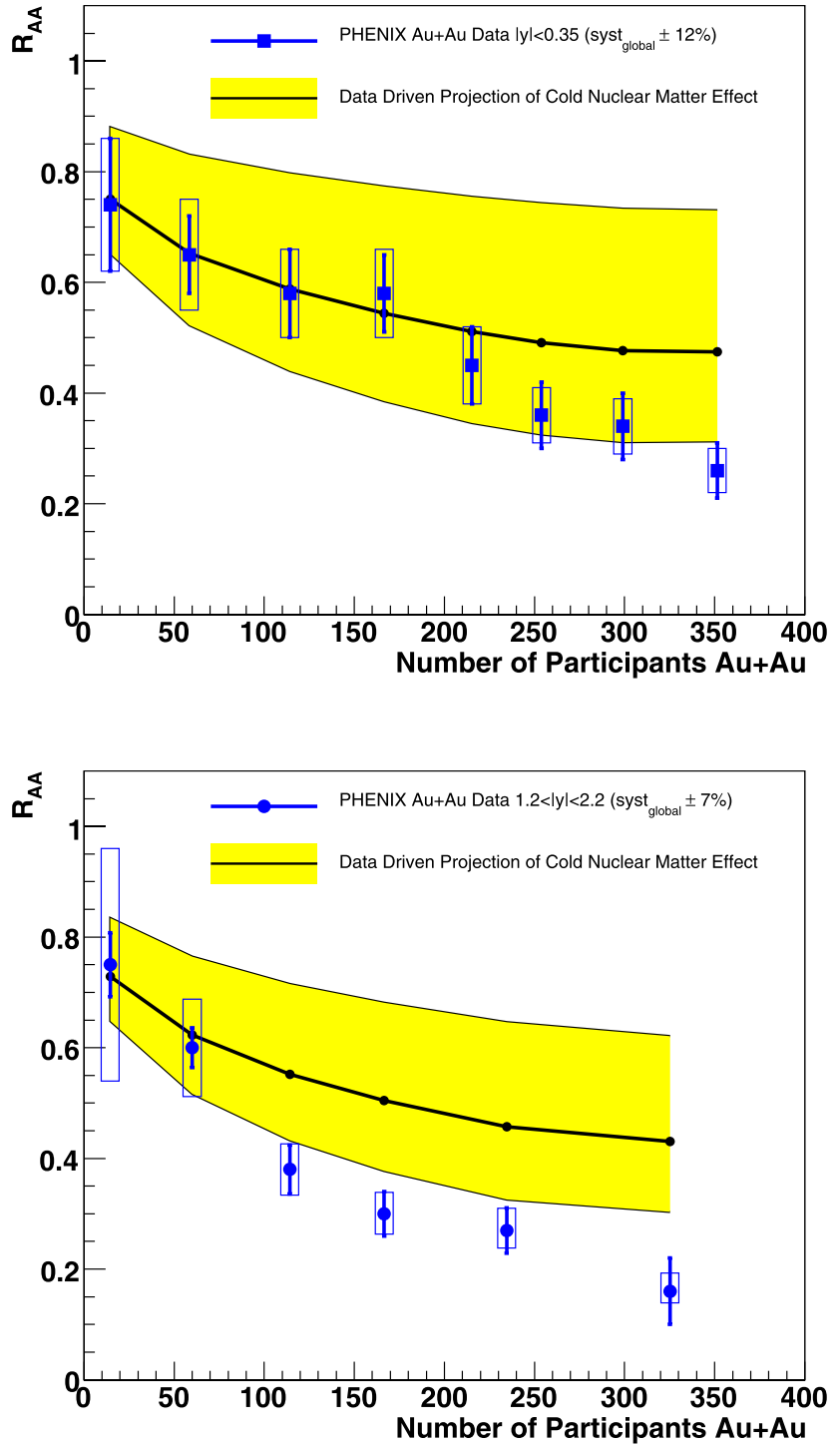

Fig. 4.1 (Color online) $R_{\mathrm{AuAu}}$ versus centrality for mid (bottom panel) and forward rapidity (top panel), the yellow band represents the uncertainty band for the extrapolation of CNM from $R_{\mathrm{dAu}}$ using a phenomenological fit coupled to a Glauber geometric model

from the understanding of the PHENIX detector. These improvements, in addition to the reduction in statistical uncertainties and the systematic associated with extracting the $J / \psi$ signal when one has low counts will result in a reduced uncertainty band in the near future.

\section{Conclusion}

It is not yet clear whether normal density nuclear matter effects can account for the $J / \psi$ suppression seen in heavy ion collisions at RHIC. However it is clear that one has to take care when relying on models to predict the amount of modification resulting from CNM effects. For instance the applicability of nuclear shadowing models to $\mathrm{d}+$ Au collision rapidity distributions is only valid if the production model is the so called "intrinsic" mechanism. Recently other mechanisms have been shown to predict for instance the $J / \psi$ production cross section at the Tevatron with better accuracy $[14,15]$. These "extrinsic" mechanisms provide one with a very different relationship between the parton momentum fraction $x$ and the $J / \psi$ rapidity ( $y$ ) distribution. Another concern is that the Glauber based geometric models that allow one to convolute the nPDFs and centrality dependence could be the wrong approach. In contrast to model based predictions one can also apply a data driven technique to make an extrapolation to heavy ion collisions. Within the uncertainty bands propagated to the prediction in heavy ion collisions it is not clear that there is any significant suppression beyond that expected from CNM effects. It is clear that the study of cold nuclear matter effects at PHENIX as a predictor for anomalous $J / \psi$ suppression has become very important. The future thrust of this measurement is to reduce the systematic and statistical errors on the $d+$ Au nuclear modification factor using the large run- $8 \mathrm{~d}+\mathrm{Au}$ and run- 6 $\mathrm{p}+\mathrm{p}$ data sets. Reducing the systematics requires improving our understanding of the acceptance and efficiency of the detector. This is the main thrust of our current work and we plan to have the new data available soon, which should have smaller uncertainty bands in the heavy ion extrapolations of CNM effects.

\section{References}

1. T. Matsui, H. Satz, Phys. Lett. B 178, 416 (1986)

2. D. de Florian, R. Sassot, Phys. Rev. D 69, 074028 (2004). hep-ph/0311227

3. K.J. Eskola, V.J. Kolhinen, R. Vogt, Nucl. Phys. A 696, 729 (2001). hep-ph/0104124

4. M.L. Miller, K. Reygers, S.J. Sanders, P. Steinberg, Annu. Rev. Nucl. Part. Sci. 57, 205 (2007). nucl-ex/0701025

5. A. Adare et al. (PHENIX, Collaboration), Phys. Rev. C 77, 024912 (2008)

6. A. Adare et al. (PHENIX, Collaboration), Phys. Rev. Lett. 98, 232301 (2007)

7. A. Adare et al. (PHENIX, Collaboration), Phys. Rev. Lett. 101, $122301(2008)$

8. S.S. Adler et al. (PHENIX, Collaboration), Phys. Rev. Lett. 96, 012304 (2006)

9. R. Vogt, Cold nuclear matter effects on quarkonia, in RHIC \& AGS Annual Users' Meeting, 2008. www.bnl.gov/rhic_ags/ users_meeting/Workshops/2/vogt_users08.pdf

10. K.J. Eskola, H. Paukkunen, C.A. Salgado, J. High Energy Phys. 07, 102 (2008). 0802.0139

11. S.R. Klein, R. Vogt, Phys. Rev. Lett. 91, 142301 (2003). nucl-th/0305046

12. R. Vogt, Phys. Rev. C 71, 054902 (2005). hep-ph/0411378

13. R. Granier de Cassagnac, J. Phys. G 34, S955 (2007). hep-ph/0701222

14. A.M. Rakotozafindrabe, Shadowing effects and $J / \psi$ production at $p_{T} \neq 0$, in Hot Quarks, 2008. hq2008.bnl.gov/talks/ 20-Wednesday/Morning/Andry_RakotozafindrabeHQ08.pdf

15. J.P. Lansberg, H. Haberzettl, 0806.4001 (2008) 